\title{
Reliability Analysis for Main Engine Control of CFM56-3 Engine
}

\author{
Jiwei $\mathrm{Xu}{ }^{1,}$, , Ming Zhao ${ }^{2, b}$ \\ ${ }^{1}$ Aviation Engineering Institution, Civil Aviation Flight University of China, Guanghan Sichuan \\ 618307, China \\ ${ }^{2}$ Aircraft Repair and Overhaul Plant, Civil Aviation Flight University of China, Guanghan Sichuan \\ 618307, China \\ aaei-base@qq.com, ${ }^{b} 184482839 @ q q . c o m$.
}

Keywords: Reliability Analysis, Main Engine Control, Likelihood Value Test.

\begin{abstract}
The MEC is the core component of the engine control system, and influences the system performance and flight safety. A Likelihood Value (LKV) test is used to determine the rank of distributions for raw data set of MEC, and the Maximum Likelihood Estimation (MLE) is used for estimating the parameters of the chosen distribution. The reliability analysis of MEC part life that not only helps to improve the aircraft safety level, but also to provide a theoretical basis for planning reasonable maintenance schedules and reducing maintenance costs.
\end{abstract}

\section{Introduction}

The CFM56-3 is a high bypass, dual rotor, axial flow engine which supplies thrust for the Boeing 737- 300/-400/-500 aircraft, and the power to operate these Electric, Hydraulic and Pneumatic systems.[1]

The main engine control (MEC) is attached to the fuel pump at approximately the 8 o'clock position on the aft side of the accessory gearbox (AGB), The MEC is hydro-mechanical and uses fuel operated servo valves to operate, which controls fuel flow, variable stator vane position, and variable bleed valve position. It is basically a speed governor which senses engine speed (RPM) and adjusts the fuel flow as necessary to maintain the desired speed set by the thrust lever.

The MEC performs the following functions:

(1) Controls engine speed by metering fuel to the engine fuel nozzles during all modes of operation.

(2) Automatically schedules fuel flow to maintain the thrust lever speed setting and establishes the maximum safe fuel flow limit under any operating condition.

(3) Schedules variable stator vane (VSV) position and directs fuel pump high pressure fuel (PF) to the VSV actuators to position the vanes as the schedule changes.

(4) Schedules variable bleed valve (VBV) position and directs fuel pump high pressure fuel (PF) to the VBV hydraulic actuator to position the valves as a function of the VSV schedule.

(5) Schedules the position of turbine clearance air valves by the output of signal pressures (TC1 and TC2) which are a function of engine speed (N2).

(6) Initiates turbine clearance control timer sequencing by supplying TC3 signal pressure to the timer when core engine speed reaches a specific rpm during initial acceleration to takeoff power.

(7) Provides positive fuel shutoff operation independent of power lever position.

(8) Defines and regulates MEC's own operating pressures.

MEC is the core component of the engine control system, and its status will affect system performance and aircraft flying safety directly. The distribution model of the MEC part life is determined by reliability analysis, which not only helps to improve the aircraft safety level, but also to provide a theoretical basis for planning reasonable maintenance schedules and reducing maintenance costs. 


\section{Line \& Shop Reliability Data Analysis}

\section{Data Collection.}

From 2010 to 2013, the life data of MEC for a specific airline is shown in the table 1[2], includes exact failure, suspension and interval failure. (Exact failure time is from the new to failure, interval failure is from overhaul to failure, and suspension time is engine working hours to statistic time point. [3])

Table 1 MEC failure time statistical

\begin{tabular}{cccccccc}
\hline Life/(hours) & F/S & Life/hours & F/S & Life/hours & F/S & Life/hours & F/S \\
\hline 115 & F & 750 & F & 1841 & F & 3719 & F \\
202 & F & 840 & F & 1905 & F & 4017 & F \\
305 & F & 935 & F & 1986 & F & 4317 & F \\
352 & S & 1043 & F & 2176 & F & 4782 & F \\
425 & F & 1213 & F & 2300 & S & 5000 & S \\
480 & F & 1318 & F & 2560 & F & 5211 & F \\
525 & F & 1435 & F & 2736 & F & 5447 & F \\
616 & F & 1540 & S & 2950 & S & 5800 & S \\
630 & F & 1556 & F & 3217 & F & 6100 & S \\
700 & F & 1795 & F & 3505 & F & 6400 & S \\
\hline
\end{tabular}

F-Failure, S- Suspension

\section{Selection of Distribution Model}

ReliaSoft's WEIBULL++ Distribution Wizard is used to perform a Likelihood Value (LKV) test to determine the rank of distributions for a raw data set. The Likelihood Value (LKV) test, which computes the value of the log-likelihood function given the parameters of the distribution [4].

Table 2 Rank of distributions

\begin{tabular}{ccc}
\hline Distribution & LKV & Ranking \\
\hline 1P-Exponential & -287.1 & 8 \\
2P-Exponential & -286.754 & 1 \\
Normal & -298.418 & 9 \\
Lognormal & -286.592 & 4 \\
2P-Weibull & -287.1 & 7 \\
3P-Weibull & -286.042 & 2 \\
Gamma & -287.056 & 5 \\
G-Gamma & -286.512 & 3 \\
Logistic & -299.04 & 10 \\
Log-logistic & -286.859 & 6 \\
Gumbel & -343.17 & 11 \\
\hline
\end{tabular}

The models will be ranked according to how well they fit the data, with rank 1 being the best fit. In the case, the 2P-exponential distribution is the suggested model. The exponential distribution is a commonly used distribution in reliability engineering, which is a special case of the Weibull distribution. The exponential distribution is used to model the behavior of units that have a constant failure rate (or units that do not degrade with time or wear out).

\section{Hypotheses Testing}

In order to verify the rationality of the assumed distribution function, Kolmogorov Smirnov (K-S) test method is used. K-S test is a nonparametric goodness-of-fit test (GOF), and K-S single sample test is mainly used to test whether or not the actual distribution of sample data is consistent with the specified theoretical distribution. It is more accurate than $\chi^{2}$ test, and more suitable for small sample situation [5]. 
The Modified Kolmogorov-Smirnov (K-S) test returns the probability that the respective critical value (DC) is less than the calculated statistic value (D). The calculated statistic value is the difference between the observed probability and the predicted probability.

The results return the computed values of the following: $\mathrm{P}\left(D_{C}<D\right)=0.0000000548 \%$.

Low probability values, close to 0 , indicate that there is not a significant difference between the theoretical distribution and the data set.

\section{Analytical Calculation for Characteristic Quantity of Distribution Model}

Analysis Method uses the maximum likelihood estimation (MLE) for estimating the parameters of the chosen distribution. Rank Method uses the Median Ranks (MED), Confidence bounds Method uses the Fisher Matrix (FM). Calculation results show on Table 3:

Table 3 Distribution model parameters estimate

\begin{tabular}{cc}
\hline Parameter & Value \\
\hline$\lambda$ & $3.63 \mathrm{e}^{-4}$ \\
$\gamma$ (hours) & 115
\end{tabular}

Where $\gamma$ is the location parameter, if positive, shifts the beginning of the distribution by a distance of $\gamma$ to the right of the origin, signifying that the chance failures start to occur only after $\gamma$ hours of operation, and cannot occur before. The scale parameter is $\frac{1}{\lambda}$.

the reliability function of the two-parameter exponential distribution is given by:

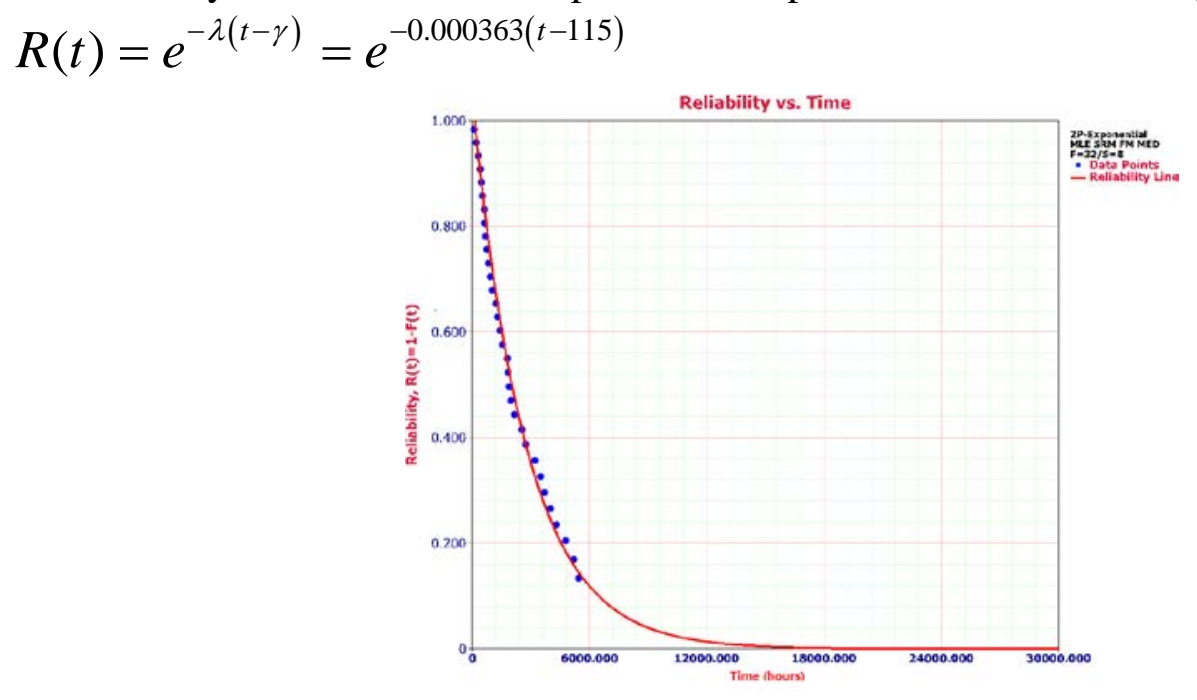

Fig. 1 Reliability vs. Time plot

The Reliability vs. Time plot shows the reliability values over time, capturing trends in the product's failure behavior.

According to MSG-3 suggestion, the overhaul interval of MEC is characteristic life:

Characteristic Life $=$ Reliable Life $\mathrm{t}(\mathrm{R}=0.368)=2754.8$ Hours

The mean time to failure (MTTF) is given by:

$\bar{T}=\int_{\gamma}^{\infty} t \cdot f(t) d t=\gamma+\frac{1}{\lambda}=2868.5$ hours

The 2-parameter exponential probability density function (pdf) is given by:

$$
f(t)=\lambda e^{-\lambda(t-\gamma)}=0.000363 e^{-0.000363(t-115)}
$$

The exponential pdf has no shape parameter, as it has only one shape.

The distribution starts at $t=\lambda$, when the level of $f(t=\gamma)=\lambda$ and decreases there after exponentially and monotonically $t$ as increases beyond $\lambda$ and is convex as $t \rightarrow \infty, f(t) \rightarrow 0$. 


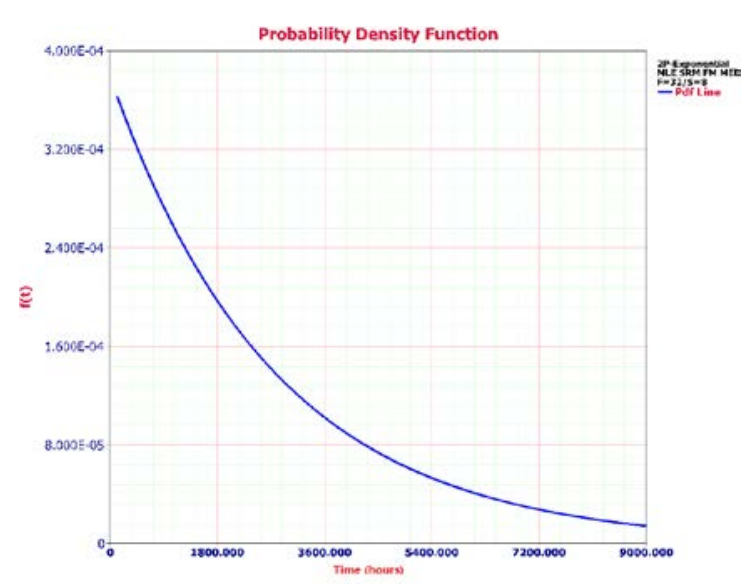

Fig. 2 Probability Density Function (pdf) plot

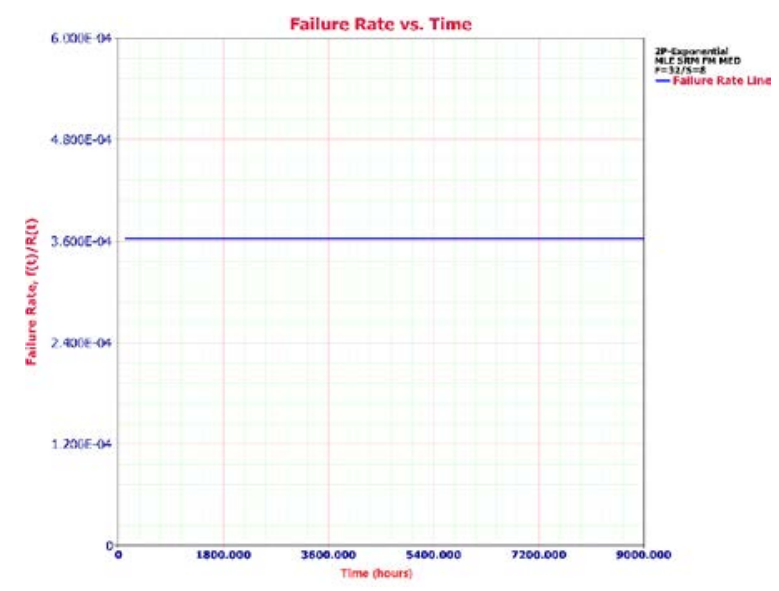

Fig.3 Failure Rate vs. Time plot

The pdf plot shows the probability density function of the data over time, allowing you to visualize the distribution of the data set.

The exponential failure rate function is:

$$
\lambda(t)=\lambda=\text { constant }
$$

Through the Failure Rate vs. Time plot analysis, the failure rate basically close to constant. This means that MEC do not degrade with time or wear out, it can be considered a random failure.

\section{Summary}

Reliability analysis is mainly used for:

If MEC using time and the average daily flight hours are known, MEC spare quantity is easily predicted based on the above analysis.

The MEC must deliver to overhaul per 6000 flight hours overhaul before. MEC failure occurred more frequently based on line experience, overhaul time interval can be adjust to2800 hours and using the "on condition" maintenance policy.

The analysis result could be as importance reference for establishing fault tree of engine fuel system.

\section{References}

[1] CFMI Customer Training Services. CFM56-3 line maintenance training manual. Cincinnati, 2012, p.10,100-102

[2] Guihuo Luo, Jixin Pu. CFM56-3 engine MEC reliability analysis. Journal of Nanjing University of Aeronautics \&. Astronautics. Vol.33 (2001) No. 1, p. 13-16.

[3] Huairui Guo, Ferenc Szidarovszky, Pengying Niu. Estimating Component Reliabilities from Incomplete System Failure Data. 2013 Reliability and Maintainability Symposium. Orlando, 28-31 Jan. 2013, p. 1-6.

[4] ReliaSoft Corporation. Weibull++/ALTA 9 User’s Guide. Tucson, p. 18.

[5] Guofang He, Hai Xu. Reliability Data Analysis and Collection. National Defense Industry Press, 1995, p. 231. 